Research Paper

\title{
MicroRNA-101-3p advances cisplatin sensitivity in bladder urothelial carcinoma through targeted silencing $\mathrm{EZH} 2$
}

\author{
Bo Li1 ${ }^{1}$, Dalong $\mathrm{Xie}^{2}$, Hui Zhang ${ }^{1 凶}$ \\ 1. Department of Urinary surgery, Shengjing Hospital, China Medical University, Shenyang, Liaoning, China \\ 2. Department of Anatomy, College of Basic Medicine, China Medical University, Shenyang, Liaoning, China \\ $\triangle$ Corresponding author: Hui Zhang, Department of Urinary surgery, Shengjing Hospital, China Medical University, No.36 Sanhao Street, Heping Area, \\ Shenyang, 100004, China. Phone/Fax: +86-24-96615; E-mail address: zhangh10@sj-hospital.org \\ (c) Ivyspring International Publisher. This is an open access article distributed under the terms of the Creative Commons Attribution (CC BY-NC) license \\ (https://creativecommons.org/licenses/by-nc/4.0/). See http://ivyspring.com/terms for full terms and conditions.
}

Received: 2019.01.14; Accepted: 2019.04.10; Published: 2019.06.02

\begin{abstract}
Objective: Chemotherapy is a major therapeutic method for bladder urothelial carcinoma (BUC), which can effectively improve the prognosis of BUC patients, but the chemoresistance often leads to chemotherapy failure. This study will research the regulatory roles and molecular mechanism of miR-101-3p in BUC chemoresistance.

Materials and Methods: The quantitative real-time PCR was used to detect the expression of miRNA-101-3p and EZH2. The proliferation and chemoresistance were analyzed by CCK8 assay. Luciferase reporter assay was used to verify the combination between miR-101-3p and EZH2. Protein expression was detected by Western blotting. Flow cytometry was used to examine apoptosis rate.

Results: The miR-101-3p expression was down-regulated in cisplatin (CDDP) resistant BUC cell line (T24/CDDP) and tissues, and was positively related to sensitivity of BUC to CDDP. In T24/CDDP cells, the up-regulation of miR-101-3p decreased the half maximal inhibitory concentration (IC50) to CDDP, depressed the expression of MRPI protein, promote the CDDP-induced cytotoxicity, and advanced CDDP sensitivity. A series of in vitro experiments certified the EZH2 gene was a target gene of miR-101-3p, including luciferase reporter assay, western blotting and so on. Up-regulation of $E Z H 2$ largely reversed the regulatory effects of miR-101-3p enhancement on CDDP sensitivity in T24/CDDP cells.

Conclusion: The expression of miR-101-3p is positively related to CDDP sensitivity of BUC, miR-101-3p advances sensitivity of BUC to CDDP through targeted silencing EZH2.
\end{abstract}

Key words: bladder urothelial carcinoma; miR-101-3p; chemotherapy; cisplatin; EZH2

\section{Introduction}

Bladder cancer is the most common malignant cancer in genitourinary system, bladder urothelial carcinoma (BUC) is the most common classification of bladder cancer, which was called bladder transitional cell carcinoma (BTCC) previously ${ }^{[1]}$. Chemotherapy is a major therapeutic method for BUC, including systemic and intravesical chemotherapy, which can effectively inhibit the progression of BUC and improve the prognosis of patients ${ }^{[2]}$. The resistance of cancer cells to chemotherapeutic drugs often leads to chemotherapy failure [3]. Therefore, it is urgent to clarify the mechanism of chemotherapy resistance, especially in BUC.

MicroRNAs are a class of small non-coding RNA molecules, that function in RNA silencing and post-transcriptional regulation of gene expression. MicroRNAs take part in multiform cellular and metabolic pathways, and are involved in the genesis 
of a variety of complex diseases, including cancers [4-6]. Recent literatures reported miR-101-3p was low-expressed and acted as a tumor-suppressing gene in diverse malignant cancers, including bladder cancer [7-10]. MiR-101-3p inhibited the invasion and migration of bladder cancer 253J-BV cells [11]. Whether miR-101-3p is involved in chemoresistance of BUC and the underlying mechanism is still unknown.

Through bioinformatics analysis, the Enhancer of Zeste 2 Polycomb Repressive Complex 2 Subunit (EZH2) gene might be a target gene of miR-101-3p. EZH2 gene is a member of the Polycomb-group (PcG) family, is a core subunit of the polycomb repressor complex 2 (PRC2), participates in the formation of multimeric protein complexes to maintain the transcriptional repressive state of genes over successive cell generations [12]. EZH2 has abnormal expression in almost all malignant cancers [13-16].

\section{Materials and Methods}

\section{Clinical specimens}

89 cases of BUC patients were treated in Shengjing Hospital between May 2012 and Jun 2013. All BUC tissue specimens were obtained through cystoscopy for pathologist diagnosis, the patients' clinicopathological data was complete and clear. Remaining BUC specimens were stored at $-80^{\circ} \mathrm{C}$ with RNAlater Stabilization Solution (Thermo Fisher Scientific, USA) and used to detect the expression of miR-101-3p and EZH2. All patients were diagnosed as BUC for the first time, and were not treated with radiotherapy or chemotherapy before diagnosis.

After diagnosis, all BUC patients received three courses of neoadjuvant chemotherapy with cisplatin (CDDP), each course is 28 days, which consisted of CDDP treatment period (7 days) and interval period (21 days). Then, all BUC patients were examined by cystoscopy and enhanced computerized tomographic scanning (enhanced CT), and the curative effects were evaluated according to RECIST Response Evaluation Criteria. If the patients showed the complete or partial remission, they were classified into the sensitive group $(n=37)$. On the contrary, if the patients' conditions were stable or deteriorating, they were classified into the resistant group $(\mathrm{n}=52)$.

\section{Cell culture}

Human BUC T24 cell line was achieved from American Type Culture Collection (ATCC), and cultured in Dulbecco's Modified Eagle's medium (DMEM) medium which included $10 \%$ fetal bovine serum (Gibco, USA) at the condition of $37^{\circ} \mathrm{C}$ and $5 \%$ $\mathrm{CO}_{2}$. T24/CDDP cell line has resistance to CDDP and was established previously in our laboratory by inducting with low concentration of CDDP [17].

\section{Quantitative real time -PCR}

Total RNA was extracted from BUC tissues and cells using Trizol reagent. FastKing One Step RT-qPCR Kit (TianGen, China) was applied to examine the expression of miR-101-3p and EZH2 through the 7500 Real-Time PCR System (Applied Biosystems, USA) according to manufacturer's instructions. The primers of miR-101-3p were 5'CTCAACTGGTGTCGTGGAGTCGGCAATTCAGTT GAG TTCAGTTA -3' (stem-loop RT-primer), 5'GCCGAGTACAGTACTGTGA -3' (Forward primer) and 5'- CTCAACTGGTGTCGTGGA -3' (Reverse primer); primers of EZH2 were 5'GGAACAACGCGAGTCGG -3' (Forward primer) and 5'- CTGATTTTACACGCTTCCGC -3' (Reverse primer). Each reaction contained $2 \times$ FastKing One Step RT-PCR MasterMix ( $25 \mu \mathrm{L}), 25 \times$ RT-PCR Enzyme Mix $(2 \mu \mathrm{L})$, Forward Primer $(1.25 \mu \mathrm{L})$, Reverse Primer (1.25 $\mu \mathrm{L})$, RNA template $(2 \mu \mathrm{L})$ and RNase-Free $\mathrm{ddH}_{2} \mathrm{O}$ $(18.5 \mu \mathrm{L})$. The cycling condition is as follows: Stage 1 : $42^{\circ} \mathrm{C}$ for $20 \mathrm{~min}, 95^{\circ} \mathrm{Cfor} 3 \mathrm{~min}, 1$ Cycle; Stage 2: $94^{\circ} \mathrm{C}$ for $30 \mathrm{sec}, 60^{\circ} \mathrm{C}$ for $30 \mathrm{sec}, 72^{\circ} \mathrm{C}$ for $30 \mathrm{sec}, 40$ Cycles; Stage 3: $72^{\circ} \mathrm{C}$ for $50 \mathrm{~min}$. The relative expression levels of miR-101-3p and EZH2 were calculated using 2- $\Delta \Delta C T$ method after normalization with reference genes (GAPDH and U6).

\section{Transfection}

The agonist of miR-101-3p (agomiR-101-3p) and its negative control (agomiR-NC) were designed and synthesized by GenePharma Company (China). The expression vector of EZH2 (pUC-EZH2) and its negative control (pUC-NC) were designed and synthesized by Cyagen Company (China). All microRNAs and vectors were transfected with Lipofectamine 3000 (Invitrogen, USA) according to manufacturer's instructions.

\section{Cell proliferation assay}

The cells $\left(2 \times 10^{4} /\right.$ well $)$ were seeded in 96-well plate. CCK8 method was used to examine the cell viability according to manufacturer's instructions. 10 $\mu \mathrm{L}$ Cell Counting Kit-8 (Beyotime, China) was added into each well of 96-well plate, and the plate was incubated 2 hours at $37{ }^{\circ} \mathrm{C}$. The SpectraMax M5 microplate reader (Molecular Devices, USA) was applied to detect the value of optical density at 450 nm.

\section{Chemotherapy sensitivity assay}

T24 and T24/CDDP Cells were seeded into 96-well plates with 3000 cells per well. 24 hours later, CDDP (10 mg/L, $25 \mathrm{mg} / \mathrm{L}, 50 \mathrm{mg} / \mathrm{L}, 75 \mathrm{mg} / \mathrm{L}, 100$ $\mathrm{mg} / \mathrm{L}$ ) was used to treat the cells [17]. The cell viability was examined by CCK 8 method after 48 hours. The dose-response curve was charted and the half 
maximal inhibitory concentration (IC50) was counted according to these data ${ }^{[18]}$.

\section{Flow cytometry assay}

The apoptosis detection was finished with Annexin V-FITC apoptosis detection kit (Biosea, China) through the Flow cytometry according to manufacturer's instructions. The results were analyzed using Diva 8.0 software (Becton Dickinson, USA). Cells in the right lower quadrant were regarded as apoptosis.

\section{Luciferase reporter assay}

The target genes of miR-101-3p was predicted by online bioinformatics software TargetScan 7.1. The luciferase reporter vectors (EZH2-M, containing mutant binding site and EZH2-W, containing wild-type binding site) were designed and synthesized by the Genescript company (China). The constructed vectors and microRNAs were cotransfected into HEK 293 cells respectively. The luciferase activity was examined by Dual Luciferase Reporter Gene Assay Kit (Beyotime, China) 48 hours later according to manufacturer's instructions.

\section{Western blotting}

Protein was extracted from T24/CDDP cells and determined the concentration with BCA Protein Assay Kit (TianGen, China) according to manufacturer's instructions. Protein samples $(20 \mathrm{mg})$ were handled with SDS-PAGE gels electrophoresis and transferred to PVDF membranes. The PVDF membrane was first hybridized with EZH2 or Multidrug Resistance Associated Protein 1 (MRP1) antibody (4905 and 72202, Cell Signaling Technology, USA), and then hybridized with secondary antibody. Then, the PVDF membrane was incubated with Pro-light HRP Chemiluminescent Kit (TianGen, China), and photographic images were taken. The intensity of the indicated bands was quantified by Image J (National Institutes of Health, USA) and normalized to the reference standard (GAPDH gene).

\section{Statistical analysis}

Each experiment was repeated five times, the result data were presented as means \pm SD and analyzed with SPSS 23.0 software (IBM, USA). Differences were assessed by one-way analysis of variance (ANOVA) in nonparametric statistics and Student's unpaired t-test. A $P<0.05$ indicated statistical significance.

\section{Results}

\section{The expression of $\mathrm{miR}-101-3 \mathrm{p}$ is positively related to CDDP sensitivity of BUC}

The expression of miR-101-3p in resistant group was down-regulated compared with sensitive group (Fig. 1A, $P<0.05$ ). And, the IC50 of T24/CDDP cell line to CDDP was $81.17 \pm 7.33 \mathrm{mg} / \mathrm{L}$, T24/CDDP cell line showed much lower sensitivity than T24 cell line which IC50 to CDDP was $26.21 \pm 3.14 \mathrm{mg} / \mathrm{L}$ (Fig. 1B, $P<0.05)$. The expression of miR-101-3p in T24/CDDP cells was much lower than that in T24 cells (Fig. 1C, $P<0.05)$. These findings reveal the expression of miR-101-3p is positively related to CDDP sensitivity of BUC, miR-101-3p plays an important role in chemoresistance of BUC to CDDP.

\section{Up-regulation of miR-101-3p advances sensitivity of T24/CDDP cells to CDDP}

Transfection with agomiR-101-3p up-regulated the expression of miR-101-3p in T24/CDDP cells. The miR-101-3p enhancement decreased the IC50 of T24/CDDP cells to CDDP from $81.06 \pm 7.09 \mathrm{mg} / \mathrm{L}$ to $38.72 \pm 3.25 \mathrm{mg} / \mathrm{L}(P<0.05$, Fig. $2 \mathrm{~A})$, and inhibited the expression of MRP1 protein in T24/CDDP cells $(P<0.05$, Fig. 2B).

Furthermore, in the T24/CDDP cells treating with CDDP $(10 \mathrm{mg} / \mathrm{L})$, the miR-101-3p enhancement depressed cell viability (Fig.2C, $P<0.05)$, and promoted cell apoptosis (Fig. 2D, $P<0.05$ ).
A

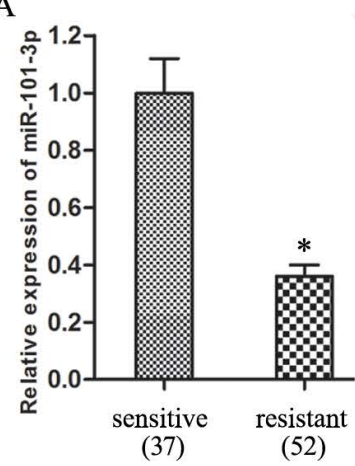

B

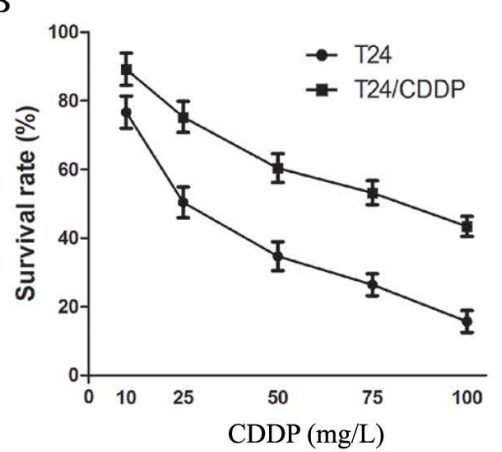

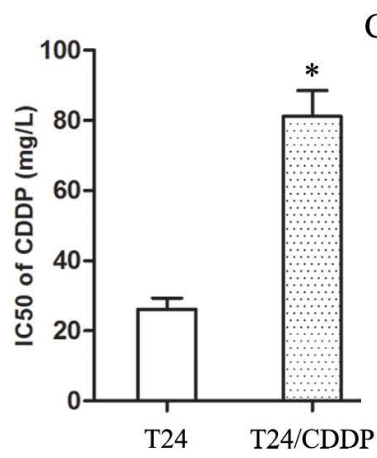

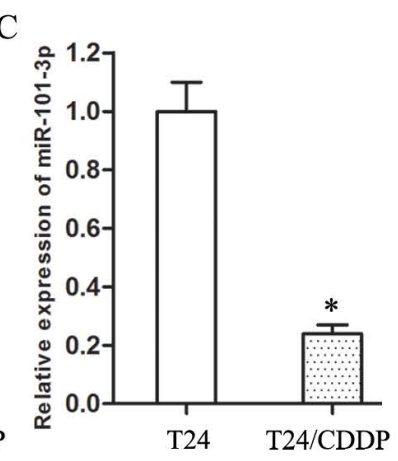

Figure 1. The expression of miR-101-3p is positively related to CDDP sensitivity of BUC. A: The expression of miR-101-3p in BUC patients treated with CDDP. B: The dose-response curve and IC50 of CDDP in T24 and T24/CDDP cells. C: The expression of miR-101-3p in T24 and T24/CDDP cells. * $P<0.05$. 

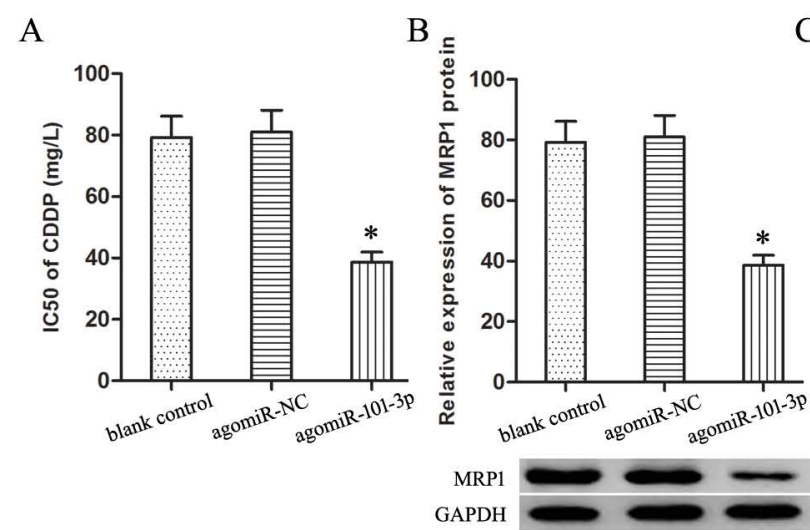

C

$\mathrm{D}$
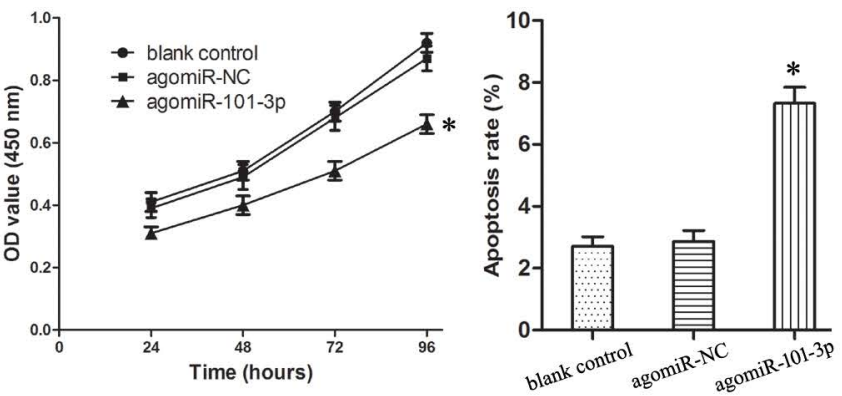

Figure 2. Up-regulation of miR-101-3p advances sensitivity of T24/CDDP cells to CDDP. A: The IC50 of CDDP in T24/CDDP cells. B: The expression of MRPI protein in T24/CDDP cells. C: The cell viability of T24/CDDP cells under treating with CDDP (10 mg/L). D: The cell apoptosis of T24/CDDP cells under treating with CDDP $(10 \mathrm{mg} / \mathrm{L}) . * P<0.05$.

\section{EZH2 gene is a target gene of miR-101-3p in BUC cells}

Through online bioinformatics software TargetScan 7.1, two potential binding sites of miR-101-3p was predicted in the 59-65 bp and 114-121bp of EZH2 3'-UTR (Fig. 3A). Luciferase reporter assay proved miR-101-3p could specifically combine with wild-type binding site to restrain the luciferase activity (Fig. 3B, $P<0.05$ ). And, miR-101-3p enhancement depressed obviously the expression of EZH2 protein in T24/CDDP cells (Fig. 3C, $P<0.05$ ). Moreover, the EZH2 expression in T24/CDDP cell line and resistant group specimens was much higher than that in T24 cell line and sensitive group specimens respectively (Fig. 3D,E, $P<0.05$ ). And, the EZH2 expression showed an inverse correlation with the expression of miR-101-3p $(P<0.01)$. In addition, the co-expression patterns analysis showed a negative correlation between miR-101-3p and EZH2 in BUC tissues (Fig. 3F) $(\mathrm{r}=-0.3951, P<0.001)$. These results confirmed that miR-101-3p could bind to EZH2 and negatively regulate the expression of EZH2, and EZH2 was a target gene of miR-101-3p.

\section{EZH2 mediates the regulation of miR-101-3p on CDDP sensitivity of T24/CDDP cells}

To determine whether EZH2 mediates the regulatory effects of miR-101-3p on chemosensitivity, knockdown of EZH2 by miR-101-3p enhancement was rescued by transfection with pUC-EZH2 prior to the detection of CDDP sensitivity, MRP1 expression and cytotoxicity induced by CDDP.

Compared with agomiR-101-3p + pUC-NC group, the IC50 of agomiR-101-3p + pUC-EZH2 group to CDDP raised significantly (Fig.4A, $P<0.05$ ), and the expression of MRP1 protein increased remarkably (Fig.4B, $P<0.05$ ).
Furthermore, under treating with CDDP (10 $\mathrm{mg} / \mathrm{L})$, compared with agomiR-101-3p + pUC-NC group, the cell viability of agomiR-101-3p + pUC-EZH2 group increased (Fig.2C, $P<0.05$ ), and cell apoptosis showed a significant decline (Fig.2D, $P<0.05)$.

\section{Discussion}

It is well known that microRNAs participate in a variety of important physiological functions by regulating the expression of target genes, their abnormal expression resulted to the genesis of a variety of complex diseases, including cancers. Some recent reports discussed microRNAs take part in formation of chemoresistance of malignant cancers, including BUC [19-22].

Our study firstly found miR-101-3p was low-expressed in CDDP resistant BUC patients and cell line, revealed the miR-101-3p expression was positively related to CDDP sensitivity of BUC. CDDP interferes with DNA replication, kills the fastest proliferating tumor cells, and is used widely in chemotherapy of many malignant cancers. Following experiment validated that miR-101-3p enhancement could inhibit the IC50 of T24/CDDP cells to CDDP, and promote the cytotoxicity induced by CDDP, which proved miR-101-3p played an important role in chemoresistance of BUC to CDDP.

Furthermore, the up-regulation of miR-101-3p also depressed the expression of MRP1 protein. MRP1 belonged to the ATP-binding cassette (ABC) transporters superfamily, which participates in the molecule transportation across the cellular membranes, which can pump chemotherapeutic drugs out of cells before they work [23-25]. Together, miR-101-3p advances CDDP sensitivity through silencing MRP1 expression, but the underlying mechanism is still unknown. 
A

EZH2-M (59-65 bp): 5'-AACCUCGA CAUGACA G-3' EZH2-W (59-65 bp): 5'-AACCUCGA GUACUGU G-3' | III|| || miR-101-3p: 3'-AAUAGUGU CAUGACA U-5' I I I I I I EZH2-W (114-121 bp): 5'-UUUGCAAA GUACUGU A-3' EZH2-M (114-121 bp): 5'-UUUGCAAA CAUGACA A-3'

C

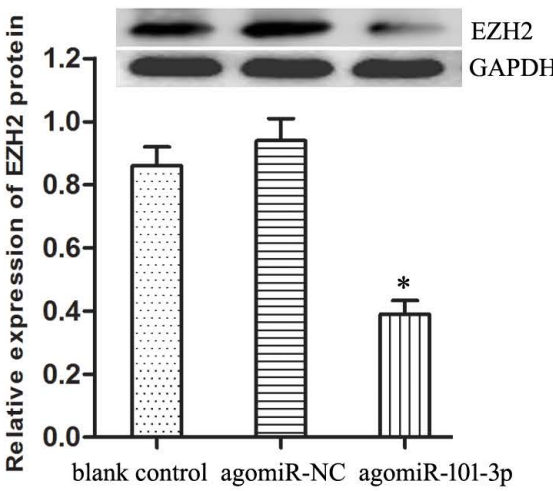

F

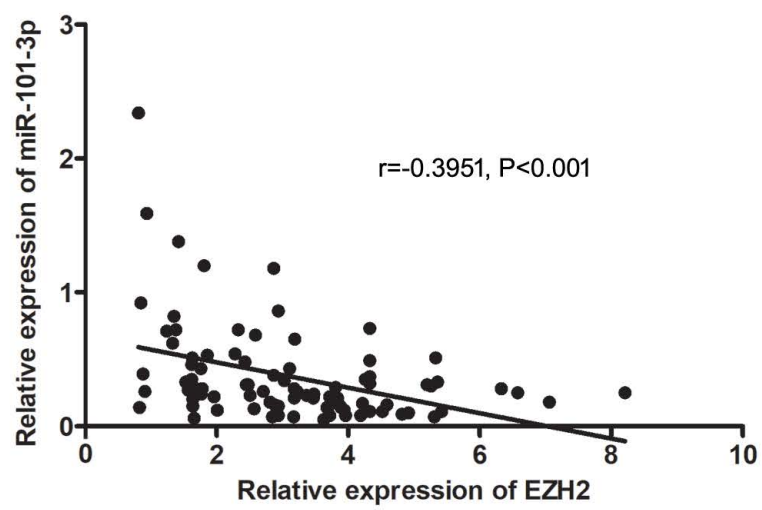

$\mathrm{B}$

D
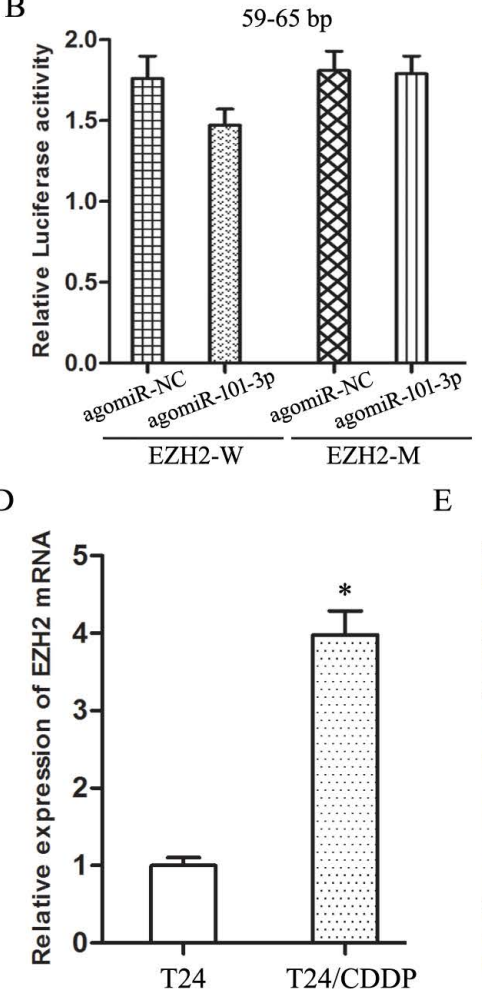

114-121 bp

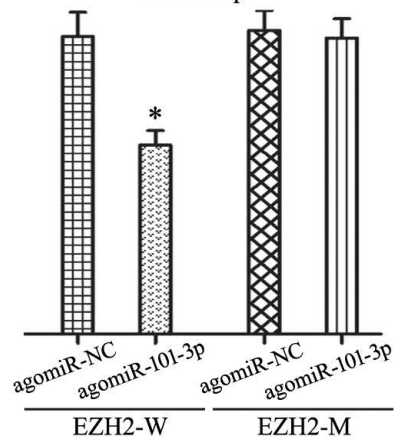

$\mathrm{E}$

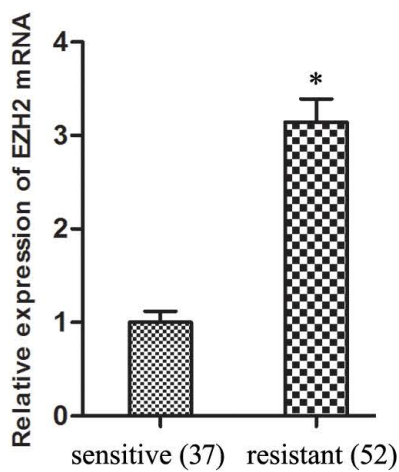

Figure 3. EZH2 gene is a target gene of miR-101-3p in BUC cells. A: The binding sites of miR-101-3p in the 3' UTR of EZH2. B: The effect of agomiR-101-3p on the luciferase activity of EZH2-W and EZH2-M. C: The expression of EZH2 protein in T24/CDDP cells. D: The expression of EZH2 mRNA in T24 and T24/CDDP cells. E: The expression of EZH2 mRNA in BUC patients treated with CDDP. F: The co-expression patterns analysis between miR-101-3P and EZH2 in BUC tissues. * $P<0.05$.
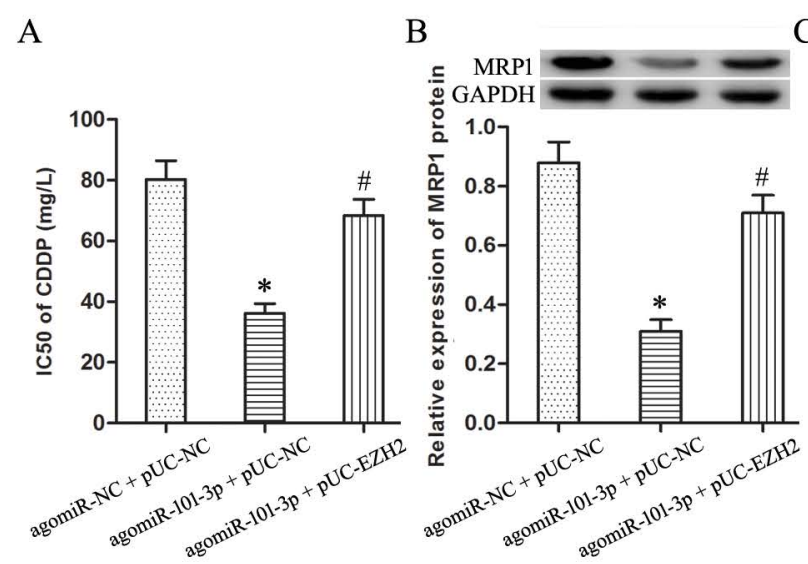

$\mathrm{C}$

D
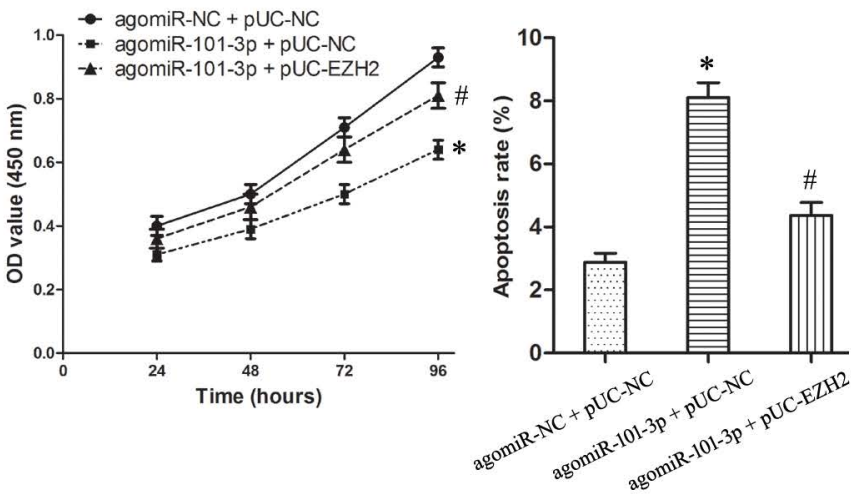

Figure 4. EZH2 mediates the regulation of miR-101-3p on CDDP sensitivity of T24/CDDP cells. A: The IC50 of CDDP in T24/CDDP cells. B: The expression of MRP1 in T24/CDDP cells. C: The cell viability of T24/CDDP cells under treating with CDDP (10 mg/L). D: The cell apoptosis of T24/CDDP cells under treating with CDDP (10 $\mathrm{mg} / \mathrm{L}) . * P<0.05$ vs agomiR-NC + pUC-NC, \# $P<0.05$ vs agomiR-101-3p + pUC-NC. 
In consideration of microRNAs paly their roles through regulating their target genes, such as miR-429 and BMI1 [26], we predicted EZH2 gene might be a target of miR-101-3p by using online bioinformatics software. And, Hou Y reported miR-101-3p could regulate the proliferation of lung squamous carcinoma via targeting EZH2 [27]. EZH2 is an activator of gene expression, acting through multiple signaling pathways in distinct cancer types. Increasing evidence indicates EZH2 acts as an oncogene and is central to initiation, growth and progression of urological cancers. A series of following gain-of-function experiments, such as luciferase reporter assay and western blotting, proved EZH2 gene was the target gene of miR-101-3p. Lv Y reported knockdown of EZH2 reduced the expression of MRP1 and advanced cisplatin sensitivity in lung adenocarcinoma [28]. To sum up, we speculate miR-101-3p might modulate the CDDP resistance of BUC cells through targeted silencing EZH2.

To verify this hypothesis, knockdown of EZH2 by miR-101-3p enhancement was rescued by transfection with pUC-EZH2. The following experiments found that up-regulation of EZH2 largely reversed the regulatory roles of miR-101-3p on CDDP resistance of T24/CDDP cells. Accordingly, EZH2 mediated the regulation of miR-101-3p on CDDP resistance in T24/CDDP cells.

In summary, the expression of miR-101-3p is positively related to CDDP sensitivity of BUC, miR-101-3p advances sensitivity of BUC on CDDP through targeted silencing EZH2. These founding might provide guidance for the clinical chemotherapy of BUC.

\section{Acknowledgements}

This work was supported by the National Nature Science Foundation of China (30901480, 81301834) and China Medical University Youth Backbone Support Program (QGZD2018051).

\section{Author Contributions}

BL carried out the in vitro studies and performed the statistical analysis. DX conceived of the study and helped to draft the manuscript. HZ participated in the study design and drafted the manuscript. All authors read and approved the final manuscript.

\section{Competing Interests}

The authors have declared that no competing interest exists.

\section{References}

1. Yousef PG, Gabril MY. An update on the molecular pathology of urinary bladder tumors. Pathol Res Pract. 2017, pii: S0344-0338(17)30807-5.
2. Kamat AM, Hahn NM, Efstathiou JA, Lerner SP, Malmström PU, Choi W, Guo CC, Lotan Y, Kassouf W. Bladder cancer. Lancet. 2016, 388(10061):2796-2810.

3. Norouzi S, Gorgi Valokala M, Mosaffa F, Zirak MR, Zamani P, Behravan J. Crosstalk in cancer resistance and metastasis. Crit Rev Oncol Hematol. 2018, 132:145-153.

4. Wu X, Chen W, Cai H, Hu J, Wu B, Jiang Y, Chen X, Sun D, An Y. MiR-216b inhibits pancreatic cancer cell progression and promotes apoptosis by down-regulating KRAS. Arch Med Sci. 2018, 14(6):1321-1332.

5. Piras S, Furfaro AL, Caggiano R, Brondolo L, Garibaldi S, Ivaldo C, Marinari UM, Pronzato MA, Faraonio R, Nitti M. microRNA-494 Favors HO-1 Expression in Neuroblastoma Cells Exposed to Oxidative Stress in a Bach1-Independent Way. Front Oncol. 2018, 8:199.

6. Shen J, Zhou W, Bi N, Song YM, Zhang FQ, Zhan QM, Wang LH. MicroRNA-886-3P functions as a tumor suppressor in small cell lung cancer. Cancer Biol Ther. 2018, 19(12):1185-1192.

7. Li XN, Wang ZJ, Ye CX, Zhao BC, Huang XX, Yang L. Circular RNA circVAPA is up-regulated and exerts oncogenic properties by sponging miR-101 in colorectal cancer. Biomed Pharmacother. 2019, 112:108611.

8. Li CY, Xiong DD, Huang CQ, He RQ, Liang HW, Pan DH, Wang HL, Wang YW, Zhu HW, Chen G. Clinical Value of miR-101-3p and Biological Analysis of its Prospective Targets in Breast Cancer: A Study Based on The Cancer Genome Atlas (TCGA) and Bioinformatics. Med Sci Monit. 2017, 23:1857-1871.

9. Jin Q, He W, Chen L, Yang Y, Shi K, You Z. MicroRNA-101-3p inhibits proliferation in retinoblastoma cells by targeting EZH2 and HDAC9. Exp Ther Med. 2018, 16(3):1663-1670.

10. Liu D, Li Y, Luo G, Xiao X, Tao D, Wu X, Wang M, Huang C, Wang L, Zeng F, Jiang G. LncRNA SPRY4-IT1 sponges miR-101-3p to promote proliferation and metastasis of bladder cancer cells through up-regulating EZH2. Cancer Lett. 2017, 388:281-291.

11. Ma Y, Luo W, Bunch BL, Pratt RN, Trump DL, Johnson CS. 1,25D3 differentially suppresses bladder cancer cell migration and invasion through the induction of miR-101-3p. Oncotarget. 2017, 8(36):60080-60093

12. Yamagishi M, Uchimaru K. Targeting EZH2 in cancer therapy. Curr Opin Oncol. 2017, 29(5):375-381.

13. Zhao Y, Ding L, Wang D, Ye Z, He Y, Ma L, Zhu R, Pan Y, Wu Q, Pang K, Hou X, Weroha SJ, Han C, Coleman R, Coleman I, Karnes RJ, Zhang J, Nelson PS, Wang L, Huang H. EZH2 cooperates with gain-of-function p53 mutants to promote cancer growth and metastasis. EMBO J. 2019, 38(5). pii: e99599.

14. Chen YT, Zhu F, Lin WR, Ying RB, Yang YP, Zeng LH. The novel EZH2 inhibitor, GSK126, suppresses cell migration and angiogenesis via down-regulating VEGF-A. Cancer Chemother Pharmacol. 2016, 77(4):757-65.

15. Wang M, Guo C, Wang L, Luo G, Huang C, Li Y, Liu D, Zeng F, Jiang G, Xiao $\mathrm{X}$. Long noncoding RNA GAS5 promotes bladder cancer cells apoptosis through inhibiting EZH2 transcription. Cell Death Dis. 2018, 9(2):238.

16. Mahmoud F, Shields B, Makhoul I, Hutchins LF, Shalin SC, Tackett AJ. Role of EZH2 histone methyltrasferase in melanoma progression and metastasis. Cancer Biol Ther. 2016, 17(6):579-91.

17. Liu Z, Zhang H. LncRNA plasmacytoma variant translocation 1 is an oncogene in bladder urothelial carcinoma. Oncotarget. 2017, 8(38):64273-64282.

18. Zhang H, Guo Y, Song Y, Shang C. Long noncoding RNA GAS5 inhibits malignant proliferation and chemotherapy resistance to doxorubicin in bladder transitional cell carcinoma. Cancer Chemother Pharmacol. 2017, 79(1):49-55

19. Zhao D, Zhang Y, Song L. MiR-16-1 Targeted Silences Far Upstream Element Binding Protein 1 to Advance the Chemosensitivity to Adriamycin in Gastric Cancer. Pathol Oncol Res. 2018, 24(3):483-488

20. Pan J, Li X, Wu W, Xue M, Hou H, Zhai W, Chen W. Long non-coding RNA UCA1 promotes cisplatin/gemcitabine resistance through CREB modulating miR-196a-5p in bladder cancer cells. Cancer Lett. 2016, 382(1):64-76.

21. Shang C, Guo $Y$, Zhang $H$, Xue YX. Long noncoding RNA HOTAIR is a prognostic biomarker and inhibits chemosensitivity to doxorubicin in bladder transitional cell carcinoma. Cancer Chemother Pharmacol. 2016 Mar, 77(3):507-13.

22. Xie D, Zhang H, Shang C. Long non-coding RNA CDKN2B antisense RNA 1 gene inhibits Gemcitabine sensitivity in bladder urothelial carcinoma. J Cancer. 2018, 9(12):2160-2166.

23. Lu JF, Pokharel D, Bebawy M. MRP1 and its role in anticancer drug resistance. Drug Metab Rev. 2015, 47(4):406-19.

24. Zhang YK, Wang YJ, Gupta P, Chen ZS. Multidrug Resistance Proteins (MRPs) and Cancer Therapy. AAPS J. 2015, 17(4):802-12. 
25. Yan X, Zhao J, Zhang R. Visfatin mediates doxorubicin resistance in human colorectal cancer cells via up regulation of multidrug resistance 1 (MDR1). Cancer Chemother Pharmacol. 2017, 80(2):395-403.

26. Peng G, Liao Y, Shen C. miRNA-429 Inhibits Astrocytoma Proliferation and Invasion by Targeting BMI1. Pathol Oncol Res. 2017, 23(2):369-376.

27. Hou Y, Li L, Ju Y, Lu Y, Chang L, Xiang X. MiR-101-3p Regulates the Viability of Lung Squamous Carcinoma Cells via Targeting EZH2. J Cell Biochem. 2017, 118(10):3142-3149.

28. Lv Y, Yuan C, Xiao X, Wang X, Ji X, Yu H, Wu Z, Zhang J. The expression and significance of the enhancer of zeste homolog 2 in lung adenocarcinoma. Oncol Rep. 2012, 28(1):147-54. 